\title{
Impact of an inclined magnetic field, heat generation/absorption and radiation on the peristaltic flow of a Micropolar fluid through a porous non-uniform channel with slip velocity
}

\author{
Ajaz Ahmad Dar ${ }^{1}$, K. Elangovan ${ }^{2}$ \\ ${ }^{1}$ Department of Mathematics, Annamalai University, India \\ ${ }^{2}$ Mathematics Section, FEAT, Annamalai University, India
}

Received: 12 August 2016, Accepted: 27 February 2017

Published online: 25 August 2017.

\begin{abstract}
Incompressible peristaltic flow of a micro-polar fluid through a permeable non-uniform channel in the vicinity of an inclined magnetic field with heat and mass transfer is investigated in the present study. Effects of heat generation, radiation and spin velocity on the fluid are also studied. Presumptions of long wavelength and low Reynolds number approximations are utilized. Mathematical expressions for axial velocity, micro-rotation speed, pressure gradient, volumetric stream rate, temperature and concentration are described in the physical area. The impact of different applicable physical parameters is dissected hypothetically and figured numerically. The outcomes got are delineated and showed graphically. The outcomes determine that the impact of Magnetic field, coupling number, micropolar parameter, slip parameter, inclination of magnetic field parameter, porosity parameter, heat generation and thermal radiation parameter is extremely protuberant in the phenomena.
\end{abstract}

Keywords: Micropolar fluid, inclined magnetic field, non-uniform channel, heat generation, radiation.

\section{Introduction}

Peristaltic pumping has remained the objective of technical and engineering exploration in the recent years. The word peristalsis derives from a Greek word "Peristaltikos" which means clutching and compressing. The peristaltic transport is peripatetic compression wave along a tube-like construction, and it comes about physiologically from neuro-muscular properties of any cylindrical smooth muscle. Peristalsis is one of the significant mechanisms for liquid transport in numerous organic frameworks. Peristalsis is an intrinsic property of various biological systems having smooth muscle tubes which transports bio-fluids by its propulsive movements. Such flows extensively emerge in the transport of urine from kidney to the bladder, chyme movement in gastrointestinal tract, movement of ovum in the female fallopian tubes, blood circulation in the small blood vessels, etc. From industrial point of view the peristaltic flows play a significant role in sanitary fluid transport, transport of corrosive fluids, a noxious liquid transport in the nuclear industry etc. To understand the peristaltic action under different normal and pathological situations, many hypothetical and investigative efforts have been made since the persuasive work of Latham[1] and Shapiro et al. [2]. Some recent investigations portraying the peristaltic system of Newtonian and non-Newtonian fluids may be said in [3]-[15]

The micropolar liquids are generally characterized as isotropic, polar liquids in which contortion of the particles is disregarded. Because of its unfussiness, the model of micropolar liquid is by and large utilized for elucidation of genuine 
liquids with internal structure. Micropolar liquid is a unique sort of non-Newtonian liquid with micro-structure which fits in with a class of liquids with non-symmetrical stress tensor alluded to as polar liquids. Physically, it suggests liquids including unevenly arranged particles suspended in a gooey medium. Such a liquid can bolster couple stresses, body couples and uncovers micro-rotational and micro-inertial properties. It is all around acknowledged that various organic liquids carry on like suspensions of deformable or unbending particles in a Newtonian liquid. Such fluids are used in examining fluid flowing in brain, liquid crystals, exotic lubricants, the flow of colloidal suspensions, animal blood, etc. The hypothesis of micropolar liquids was proposed first by Eringen [16]. Further details related to the theory and applications of micropolar fluids can be found in [17]-[21].

Convection flows emerges in numerous physical circumstances, for example, in the cooling of atomic reactors and natural heat exchange forms amongst others. The issues of magneto hydrodynamic free convective flow in a permeable medium have drawn impressive considerations of a few specialists in different investigative and innovative applications like industrial processes in metallurgy, material processing in chemical industry, nuclear engineering, industrial power engineering, plasma jet engines, pumps, generators, accelerators, magnetic control of molten iron flow and flow meters in steel industry. The investigation of flow and heat exchange of an electrically conducting micropolar liquid past a permeable plate affected by a magnetic field has pulled in light of a legitimate concern for various researchersin perspective of its applications in numerous building issues, for example, magnetohydrodynamic (MHD) generators, atomic reactors, geothermal vitality extractions and the boundary layer control in the field of aerodynamics. Remembering some particular mechanical applications, for example, polymer preparing innovation, various endeavors have been made to investigate the impact of magnetic field on boundary layer flow qualities. Bhargava et al. [22] discussed mixed convection in micropolar fluid compelled by a porous stretching sheet by means of a finite element method through uniform suction. The effect of surface conditions on the velocity, micro-rotation as well as for temperature functions has been studied. It has been observed that the micropolar fluids support in the reducing of drag forces and also act as a cooling agent. Mahmood and Waheed [23] have discussed the influence of slip velocity on the flow and heat transfer for an electrically conducting micropolar fluid over a porous stretching surface with variable heat flux in the occurrence of heat generation (absorption) and magnetic field. Rahman and Sattar [24] have investigated the MHD convective flow of a micropolar fluid through a constantly moving vertical porous plate in the occurrence of heat generation/absorption with constant suction. Recently, many researchers have investigated the heat and mass transfer in conductive-radiative MHD micropolar fluid under different conditions [25]-[32].

Inspired by the examinations and applications specified above, the aim of this examination was to consider the impacts of radiation and heat generation on micropolar fluid through non-uniform porous channel. In the present analysis slip velocity with inclined magnetic field effects are studied with fussy prominence. The highly non linear equations are simplified by adopting low Reynolds number and long wavelength approximation. The results obtained are illustrated and discussed graphically.

\section{Formulation of the problem}

Mathematical model of micro-polar through a non-uniform porous channel of uniform thickness in the presence of an inclined magnetic field is to be built to study the impact of various geometric and fluid parameters on physiological parameters. Modeling is to be done by considering an unsteady, incompressible, homogeneous and electrically conducting micro-polar fluid. The geometry of the problem is shown in Fig.(1) and is expressed mathematically as

$$
h^{\prime}\left(X^{\prime}, t^{\prime}\right)=a+\tan \alpha\left(X^{\prime}-c t^{\prime}\right)+b \sin \left(\frac{2 \pi}{\lambda}\left(X^{\prime}-c t^{\prime}\right)\right)
$$




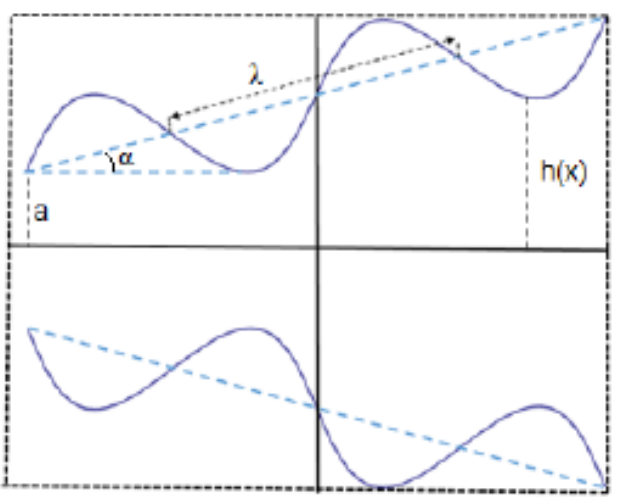

Fig. 1: The geometry of the problem

Where $a$ is half width of the of the channel at the inlet, $b$ is the amplitude of wave, $\alpha$ is the angle between the axis of the channel and the walls, $\lambda$ is the wave length, $\mathrm{c}$ is the wave velocity, $t^{\prime}$ is the time, $X^{\prime}$ and $Y^{\prime}$ represent the rectangular co-ordinates with $X^{\prime}$ measured the axis of the channel and $Y^{\prime}$ the transverse axis perpendicular to $X^{\prime}$.The accompanied transformations from the fixed frame of reference $\left(X^{\prime}, Y^{\prime}\right)$ to the wave frame of reference $\left(x^{\prime}, y^{\prime}\right)$ are given as

$x^{\prime}=X^{\prime}-c t^{\prime}, y^{\prime}=Y^{\prime}, u^{\prime}=U^{\prime}-c, v^{\prime}=V^{\prime}, w^{\prime}=W^{\prime}$.

where $\left(u^{\prime}, v^{\prime}\right)$ and $\left(U^{\prime}, V^{\prime}\right)$ are the velocity components in the wave frame and fixed frame of reference, respectively. Neglecting the body couple, the micro-inertia and viscous dissipation, the governing equations for unsteady flow of a micro-polar fluid taking into account the presence of heat generation (absorption) in the energy equation [23] are given by Conservation of mass:

$$
\frac{\partial u^{\prime}}{\partial x^{\prime}}+\frac{\partial v^{\prime}}{\partial y^{\prime}}=0
$$

Conservation of momentum:

$$
\begin{aligned}
\rho\left(\frac{\partial u^{\prime}}{\partial t^{\prime}}+u^{\prime} \frac{\partial u^{\prime}}{\partial x^{\prime}}+v^{\prime} \frac{\partial u^{\prime}}{\partial y^{\prime}}\right)= & -\frac{\partial p^{\prime}}{\partial x^{\prime}}+(\mu+k)\left(\frac{\partial^{2} u^{\prime}}{\partial x^{\prime 2}}+\frac{\partial^{2} u^{\prime}}{\partial y^{\prime 2}}\right)+k \frac{\partial g^{\prime}}{\partial y^{\prime}} \\
& -\sigma B_{0}^{2} \cos \Theta\left(\cos \Theta\left(u^{\prime}+c\right)-v^{\prime} \sin \Theta\right)-\frac{\mu}{K_{1}^{\prime}}\left(u^{\prime}+1\right) .
\end{aligned}
$$

Conservation of angular momentum:

$$
\begin{aligned}
\rho\left(\frac{\partial v^{\prime}}{\partial t^{\prime}}+u^{\prime} \frac{\partial v^{\prime}}{\partial x^{\prime}}+v^{\prime} \frac{\partial v^{\prime}}{\partial y^{\prime}}\right)= & -\frac{\partial p^{\prime}}{\partial y^{\prime}}+(\mu+k)\left(\frac{\partial^{2} v^{\prime}}{\partial x^{\prime 2}}+\frac{\partial^{2} v^{\prime}}{\partial y^{\prime 2}}\right)-k \frac{\partial g^{\prime}}{\partial x^{\prime}} \\
& +\sigma B_{0}^{2} \sin \Theta\left(\cos \Theta\left(u^{\prime}+c\right)-v^{\prime} \sin \Theta\right)-\frac{\mu}{K_{1}^{\prime}}\left(v^{\prime}\right) \\
\rho j^{\prime}\left(\frac{\partial g^{\prime}}{\partial t^{\prime}}+u^{\prime} \frac{\partial g^{\prime}}{\partial x^{\prime}}+v^{\prime} \frac{\partial g^{\prime}}{\partial y^{\prime}}\right)= & -2 k g^{\prime}+\gamma\left(\frac{\partial^{2} g^{\prime}}{\partial x^{\prime 2}}+\frac{\partial^{2} g^{\prime}}{\partial y^{\prime 2}}\right)+k\left(\frac{\partial v^{\prime}}{\partial x^{\prime}}-\frac{\partial u^{\prime}}{\partial y^{\prime}}\right)
\end{aligned}
$$

Energy:

$$
\rho c_{p}\left(\frac{\partial T^{\prime}}{\partial t^{\prime}}+u^{\prime} \frac{\partial T^{\prime}}{\partial x^{\prime}}+v^{\prime} \frac{\partial T^{\prime}}{\partial y^{\prime}}\right)=K\left(\frac{\partial^{2} T^{\prime}}{\partial x^{\prime 2}}+\frac{\partial^{2} T^{\prime}}{\partial y^{\prime 2}}\right)-\frac{\partial q_{r}}{\partial y^{\prime}}-Q^{\prime}\left(T^{\prime}-T_{0}\right)
$$


Concentration:

$$
\left(\frac{\partial C^{\prime}}{\partial t^{\prime}}+u^{\prime} \frac{\partial C^{\prime}}{\partial x^{\prime}}+v^{\prime} \frac{\partial C^{\prime}}{\partial y^{\prime}}\right)=D_{m}\left(\frac{\partial^{2} C^{\prime}}{\partial x^{\prime 2}}+\frac{\partial^{2} C^{\prime}}{\partial y^{\prime 2}}\right)+\frac{D_{m} K_{T}}{T_{m}}\left(\frac{\partial^{2} T^{\prime}}{\partial x^{\prime 2}}+\frac{\partial^{2} T^{\prime}}{\partial y^{\prime 2}}\right)
$$

where $u^{\prime}$ and $v^{\prime}$ are the velocity components in the $x^{\prime}$ and $y^{\prime}$ directions, respectively, $\rho$ is the density of the fluid, $p^{\prime}$ is the pressure, $\mu$ is the viscosity constant of the classical fluid dynamics, $\sigma$ is the electrical conductivity, $B_{0}$ is the strength of the magnetic field, $\Theta$ is inclination angle of the magnetic field parameter, $g^{\prime}$ is the microrotation velocity, $j^{\prime}$ is the micro-inertia density, $k$ is the microrotation parameter and $\gamma$ is the spin-gradient viscosity coefficient respectively. $T^{\prime}$ is temperature, $C^{\prime}$ is the concentration, $c_{p}$ is specific heat at constant pressure, $q_{r}$ is the radiation heat flux, $K$ is thermal conductivity, $Q^{\prime}$ is heat generation, $D_{m}$ is the coefficient of mass diffusivity and $K_{T}$ is the thermal diffusion ratio.

Now we introduce the non-dimensional variables and parameters as follows

$$
\begin{array}{r}
x=\frac{x^{\prime}}{\lambda}, y=\frac{y^{\prime}}{a}, u=\frac{u^{\prime}}{c}, v=\frac{v^{\prime}}{\delta c}, \delta=\frac{a}{\lambda}, D_{a}=\frac{K_{1}^{\prime}}{a^{2}}, t=\frac{c t^{\prime}}{\lambda}, p=\frac{p^{\prime} a^{2}}{c \lambda \mu}, j=\frac{j^{\prime}}{a^{2}}, g=\frac{a g^{\prime}}{c}, \\
M=\sqrt{\frac{\sigma}{\mu}} a B_{0}, R_{e}=\frac{\rho c a}{\mu}, h(x)=\frac{h^{\prime}\left(x^{\prime}\right)}{a}, t=\frac{c t^{\prime}}{\lambda}, \theta=\frac{T^{\prime}-T_{0}}{T_{1}-T_{0}}, \phi=\frac{C^{\prime}-C_{0}}{C_{1}-C_{0}}, P_{r}=\frac{\mu c_{p}}{K} \\
S_{r}=\frac{\rho D_{m} K_{T}\left(T_{1}-T_{0}\right)}{T_{m} \mu\left(C_{1}-C_{0}\right)}, S_{c}=\frac{\mu}{\rho D_{m}}, Q=\frac{Q^{\prime} a^{2}}{c_{p} \mu}, N_{r}=\frac{16 \sigma^{\prime} T_{0}^{3}}{3 k^{\prime} K} .
\end{array}
$$

Where $M$ is the Hartman number, $R_{e}$ is the Reynolds number, $\delta$ is the wave number, $D_{a}$ is the porosity parameter, $P_{r}$ is the Prandtl number, $S_{c}$ is the Schmidt number, $S_{r}$ is the Soret number, $Q$ is the heat generation parameter and $N_{r}$ is the thermal radiation parameter, $T_{m}$ is the mean temperature.

Taking after Rosseland diffusion approximation, the radiative flux $q_{r}$ for radiation is sculpted as

$$
q_{r}=-\frac{4 \sigma^{\prime}}{3 k^{\prime}} \frac{\partial T^{\prime 4}}{\partial y^{\prime}}
$$

where $\sigma^{\prime}$ denotes Stefan-Boltzman constant and $k^{\prime}$ is defined as mean absorption coefficient [33]. It is eminent that the optically thick radiation limit is pondered in this model. Supposing that the temperature variances within the flow are adequately small such that $T^{\prime 4}$ can be represented as the linear combination of temperature. This is practiced by expanding $T^{\prime 4}$ by using Taylor series about $T_{0}$ as follows

$$
T^{\prime 4}=T_{0}^{4}+4 T_{0}^{3}\left(T^{\prime}-T_{0}\right)+6 T_{0}^{2}\left(T^{\prime}-T_{0}\right)^{2}+\cdots
$$

By neglecting the higher order terms (second order onwards) in $\left(T^{\prime}-T_{0}\right)$, we arrive at

$$
T^{\prime 4} \approx-3 T_{0}^{4}+4 T_{0}^{3} T^{\prime}
$$

Differentiating Eq.(10) with respect to $y^{\prime}$ and using Eq.(12) to get

$$
\frac{\partial q_{r}}{\partial y^{\prime}}=-\frac{16 \sigma^{\prime} T_{0}^{3}}{3 k^{\prime}} \frac{\partial^{2} T^{\prime}}{\partial y^{\prime 2}}
$$


Using the non-dimensional variables and parameters from Eq.(9) in Eqs. (4)-(8), we get the modified equations as

$$
\begin{gathered}
R_{e} \delta\left(u \frac{\partial u}{\partial x}+v \frac{\partial u}{\partial y}\right)=-\frac{\partial p}{\partial x}+\frac{(\mu+k)}{\mu}\left(\delta^{2} \frac{\partial^{2} u}{\partial x^{2}}+\frac{\partial^{2} u}{\partial y^{2}}\right) \\
+\frac{k}{\mu} \frac{\partial g}{\partial y}-M^{2} \cos \Theta((u+1) \cos \Theta-v \delta \sin \Theta)-\frac{u+1}{D_{a}} \\
R_{e} \delta^{3}\left(u \frac{\partial v}{\partial x}+v \frac{\partial v}{\partial y}\right)=-\frac{\partial p}{\partial y}+\frac{(\mu+k)}{\mu} \delta^{2}\left(\delta^{2} \frac{\partial^{2} u}{\partial x^{2}}+\frac{\partial^{2} u}{\partial y^{2}}\right) \\
-\delta^{2} \frac{k}{\mu} \frac{\partial g}{\partial x}+M^{2} \delta \sin \Theta((u+1) \cos \Theta-v \delta \sin \Theta)-\delta^{2} \frac{v}{D_{a}} . \\
\rho a c j \delta\left(u \frac{\partial g}{\partial x}+v \frac{\partial g}{\partial y}\right)=-2 k g+\frac{\gamma}{a^{2}}\left(\delta^{2} \frac{\partial^{2} g}{\partial x^{2}}+\frac{\partial^{2} g}{\partial y^{2}}\right)+k\left(\delta^{2} \frac{\partial v}{\partial x}-\frac{\partial u}{\partial y}\right) \\
R_{e} \delta\left(u \frac{\partial \theta}{\partial x}+v \frac{\partial \theta}{\partial y}\right)=\frac{1}{P_{r}}\left(\delta^{2} \frac{\partial^{2} \theta}{\partial x^{2}}+\frac{\partial^{2} \theta}{\partial y^{2}}\right)+\frac{N_{r}}{P_{r}} \frac{\partial^{2} \theta}{\partial y^{2}}-Q \theta \\
R_{e} \delta\left(u \frac{\partial \phi}{\partial x}+v \frac{\partial \phi}{\partial y}\right)=\frac{1}{S_{c}}\left(\delta^{2} \frac{\partial^{2} \phi}{\partial x^{2}}+\frac{\partial^{2} \phi}{\partial y^{2}}\right)+S_{r}\left(\delta^{2} \frac{\partial^{2} \theta}{\partial x^{2}}+\frac{\partial^{2} \theta}{\partial y^{2}}\right)
\end{gathered}
$$

Using the long wavelength approximation and neglecting the wave number along with low Reynolds number, one can find from equations (14)-(18) that

$$
\begin{gathered}
(1-N) \frac{\partial p}{\partial x}=\frac{\partial^{2} u}{\partial y^{2}}+N \frac{\partial g}{\partial y}-(1-N)\left(\frac{1}{D_{a}}+M^{2} \cos ^{2} \Theta\right)(u+1) \\
\frac{\partial p}{\partial y}=0 \\
2 g=-\frac{\partial u}{\partial y}+\left(\frac{2-N}{m^{2}}\right) \frac{\partial^{2} g}{\partial y^{2}} \\
\left(1+N_{r}\right) \frac{\partial^{2} \theta}{\partial y^{2}}-P_{r} Q \theta=0 \\
\frac{\partial^{2} \phi}{\partial y^{2}}+S_{c} S_{r} \frac{\partial^{2} \theta}{\partial y^{2}}=0
\end{gathered}
$$

where $N=\frac{k}{(\mu+k)}$ is the coupling number, $(0 \leq N \leq 1)$ and $m^{2}=\frac{a^{2} k(2 \mu+k)}{\gamma(\mu+k)}$ is the micro-polar parameter.

The dimensionless boundary conditions are given as

$$
\begin{gathered}
u=-1-\beta \frac{\partial u}{\partial y}, \quad \theta=0, \quad \phi=0 \quad \text { at } \quad y=h(x) \\
u=-1+\beta \frac{\partial u}{\partial y}, \quad \theta=1, \quad \phi=1 \quad \text { at } \quad y=-h(x) \\
g=0 \quad \text { at } \quad y= \pm h(x)
\end{gathered}
$$




$$
\frac{\partial u}{\partial y}=0 \quad \text { at } \quad y=0
$$

where $\beta$ is the non-dimensional slip parameter and $\mathrm{h}$ is the non-dimensional form of the upper wall of the chanel and is expressed as

$$
h=1+\frac{x \tan (\alpha)}{\delta}+\varepsilon \sin (2 \pi x)
$$

where $\varepsilon=\frac{b}{a}$ is defined as the amplitude ratio.

\section{Solution of the problem}

From Eq.(20) it is clear that $\mathrm{p}$ is independent of $\mathrm{y}$. Therefore $\frac{\partial p}{\partial x}=\frac{d p}{d x}$. Differentiating Eq.(21) with respect to $\mathrm{y}$, we get

$$
\frac{\partial^{2} u}{\partial y^{2}}=-2 \frac{\partial g}{\partial y}+\left(\frac{2-N}{m^{2}}\right) \frac{\partial^{3} g}{\partial y^{3}}
$$

Upon making use of Eq.(26) in Eq.(19), we get

$$
u=\frac{1}{(1-N)\left(M^{2} \cos ^{2} \Theta+\frac{1}{D_{a}}\right)}\left\{\left(\frac{2-N}{m^{2}}\right) \frac{\partial^{3} g}{\partial y^{3}}-(2-N) \frac{\partial g}{\partial y}-(1-N) \frac{d p}{d x}\right\}
$$

Using this value of (u) in Eq.(21) we get

$$
\frac{\partial^{4} g}{\partial y^{4}}-\left\{(1-N)\left(M^{2} \cos ^{2} \Theta+\frac{1}{D_{a}}\right)+m^{2}\right\} \frac{\partial^{2} g}{\partial y^{2}}+\frac{2 m^{2}\left\{(1-N)\left(M^{2} \cos ^{2} \Theta+\frac{1}{D_{a}}\right)\right\}}{2-N} g=0
$$

whose general solution is

$$
g=C_{1}(x) \cosh \left(A_{1} y\right)+C_{2}(x) \sinh \left(A_{1} y\right)+C_{3}(x) \cosh \left(A_{2} y\right)+C_{4}(x) \sinh \left(A_{2} y\right)
$$

where

$$
A_{1}=\frac{1}{\sqrt{2}} \sqrt{m_{1}-\sqrt{m_{1}^{2}-4 m_{2}}}
$$

and

$$
A_{2}=\frac{1}{\sqrt{2}} \sqrt{m_{1}+\sqrt{m_{1}^{2}-4 m_{2}}}
$$

Where

$$
m_{1}=(1-N)\left(M^{2} \cos ^{2} \Theta+\frac{1}{D_{a}}\right)+m^{2}, \quad m_{2}=\frac{2 m^{2}(1-N)\left(M^{2} \cos ^{2} \Theta+\frac{1}{D_{a}}\right)}{2-N} .
$$

Substituting Eq. (29) into Eq. (27), we get

$$
\begin{aligned}
u= & \frac{1}{(1-N)\left(M^{2} \cos ^{2} \Theta+\frac{1}{D_{a}}\right)}\left\{\wedge_{1}\left[C_{1}(x) \sinh \left(A_{1} y\right)+C_{2}(x) \cosh \left(A_{1} y\right)\right]\right. \\
& \left.+\wedge_{2}\left[C_{3}(x) \sinh \left(A_{2} y\right)+C_{4}(x) \cosh \left(A_{2} y\right)\right]-(1-N) \frac{d p}{d x}\right\}
\end{aligned}
$$


where

$$
\begin{aligned}
& \wedge_{1}=(2-N) A_{1}\left(\frac{A_{1}^{2}}{m^{2}}-1\right) \\
& \wedge_{2}=(2-N) A_{2}\left(\frac{A_{2}^{2}}{m^{2}}-1\right)
\end{aligned}
$$

The expressions for axial and spin velocities can be found by applying the boundary conditions from Eq.(24) into Eq.(29) and Eq.(32). The expression for axial velocity is given as

$$
u=\frac{\left(M^{2} \cos ^{2} \Theta+\frac{1}{D_{a}}-\frac{d p}{d x}\right)\left(\wedge_{2} \cosh \left(A_{2} y\right) \sinh \left(A_{1} h\right)-\wedge_{1} \cosh \left(A_{1} y\right) \sinh \left(A_{2} h\right)\right)-L \frac{d p}{d x}}{L\left(M^{2} \cos ^{2} \Theta+\frac{1}{D_{a}}\right)}
$$

where

$$
L=\left[\wedge_{1} \cosh \left(A_{1} h\right)+\beta \wedge_{1} A_{1} \sinh \left(A_{1} h\right)\right] \sinh \left(A_{2} h\right)-\left[\wedge_{2} \cosh \left(A_{2} h\right)+\beta \wedge_{2} A_{2} \sinh \left(A_{2} h\right)\right] \sinh \left(A_{1} h\right)
$$

The expression for spin velocity is given as

$$
g=\frac{(1-N)\left(M^{2} \cos ^{2} \Theta+\frac{1}{D_{a}}-\frac{d p}{d x}\right)\left(\sinh \left(A_{2} y\right) \sinh \left(A_{1} h\right)-\sinh \left(A_{1} y\right) \sinh \left(A_{2} h\right)\right)}{L}
$$

The expression for the temperature and concentration distribution can be obtained by solving Eq.(22) and Eq.(23) with the help of boundary conditions from Eq.(24). The mathematical expressions for temperature distribution $\theta$ and concentration distribution $\phi$ are respectively given below

$$
\begin{gathered}
\theta(y)=\frac{1}{2}\left(\frac{\cosh \left(s_{1} y\right)}{\cosh \left(s_{1} h\right)}-\frac{\sinh \left(s_{1} y\right)}{\sinh \left(s_{1} h\right)}\right) \\
\phi(y)=\frac{S_{C} S_{r}}{2}\left\{\frac{\sinh \left(s_{1} y\right)}{\sinh \left(s_{1} h\right)}-\frac{\cosh \left(s_{1} y\right)}{\cosh \left(s_{1} h\right)}-\frac{y}{h}+1\right\}-\frac{1}{2}\left(\frac{y}{h}-1\right)
\end{gathered}
$$

where

$$
s_{1}=\sqrt{\frac{Q P_{r}}{1+N_{r}}} .
$$

The expression for the flux $q$ in the wave frame of reference is given by

$$
q=\int_{-h(x)}^{h(x)} u(x, y) d y=\frac{2\left(M^{2} \cos ^{2} \Theta+\frac{1}{D_{a}}-\frac{d p}{d x}\right)\left(\left(\frac{\Lambda_{2}}{A_{2}}-\frac{\Lambda_{1}}{A_{1}}\right) \sinh \left(A_{1} h\right) \sinh \left(A_{2} h\right)\right)-2 L \frac{d p}{d x} h}{L\left(M^{2} \cos ^{2} \Theta+\frac{1}{D_{a}}\right)}
$$

The pressure gradient expression can be obtained from Eq.(40) and is given as

$$
\frac{d p}{d x}=\left(M^{2} \cos ^{2} \Theta+\frac{1}{D_{a}}\right)\left\{1+\frac{(2 h+q) L}{F(x)}\right\}
$$

where $\mathrm{F}(\mathrm{x})$ is given as

$$
F(x)=2\left(\frac{\wedge_{1}}{A_{1}}-\frac{\wedge_{2}}{A_{2}}\right) \sinh \left(A_{1} h\right) \sinh \left(A_{2} h\right)-2 L h
$$


The expressions for pressure rise $\Delta p$ and the frictional force $F$ over one wavelength are given by

$$
\begin{gathered}
\Delta p=\int_{0}^{1}\left(\frac{d p}{d x}\right) d x \\
F=\int_{0}^{1} h\left(-\frac{d p}{d x}\right) d x .
\end{gathered}
$$

\section{Numerical results and discussion}

In order to illustrate the effect of various embedding parameters on the axial velocity, spin velocity, pumping characteristic, temperature and concentration distribution, calculations are carried out numerically and displayed graphically through a set of figures. Fig.2 illustrates the variation of axial velocity for different physical parameters like magnetic field parameter $(\mathrm{M})$, coupling number $(\mathrm{N})$, micropolar parameter $(\mathrm{m})$, slip parameter $(\beta)$, inclination angle $(\Theta)$ of magnetic field and permeability parameter $\left(D_{a}\right)$. It is observed from Fig.2(a)-(b) that the axial velocity (u) decreases by increasing magnetic field parameter and coupling number. From Fig.2(c), it is illustrated that the axial velocity shows negligible increase in the middle of the channel with increase in micropolar parameter. The behavior of slip parameter $(\beta)$, inclination angle $(\Theta)$ of the magnetic field and permeability parameter $\left(D_{a}\right)$ on the axial velocity is quite opposite when compared with the magnetic field parameter $(\mathrm{M})$ and coupling number $(\mathrm{N})$. The axial velocity increases by increasing the slip parameter $(\beta)$, inclination angle $(\Theta)$ of the magnetic field and the permeability parameter $\left(D_{a}\right)$.

Fig.3 illustrates the distribution of spin velocity (g) for different values of physical parameters of interest. It is observed from the Fig.3(a)-(b) that the magnitude of the spin velocity decreases on one side of the channel with the increase of the magnetic field parameter $(\mathrm{M})$ and slip parameter $(\beta)$ while on the other side of the channel, spin velocity increases by increasing $(\mathrm{M})$ and $(\beta)$. It specifies that the rotation in micro-particles occurs in two opposite directions about the central line of the channel. The center of rotation gets shifted towards the channel wall by increasing the magnetic field strength. It is also observed that the rotation in the micro-particles becomes unable for higher values of the slip parameter near the channel walls. From the Fig.3(c)-(f), it is observed that the spin velocity increases on the upper side of the channel with the increase in micropolar parameter, inclination angle of the magnetic field, permeability parameter and the coupling number, whereas the opposite behavior is observed on the lower side of the channel.

The variation of temperature and concentration distribution for different values of physical parameters involved has been illustrated in Figs.4 and 5 respectively. Fig.4 illustrates the temperature distribution changes for different values of the Thermal Radiation parameter $\left(N_{r}\right)$, Prandtl number $\left(P_{r}\right)$ and Heat source/sink parameter $(Q)$. From Fig.5(a)-(c), it is observed that the temperature distribution increases by increasing the thermal radiation parameter while an opposite trend is observed by Prandtl number and Heat source/sink parameter when compared with the thermal radiation parameter. Fig.5 shows the concentration distribution variation for different values of $N_{r}, P_{r}, Q, S_{r}$ and $S_{c}$. It is observed that the concentration distribution has exactly opposite behavior when compared with the temperature distribution. That is, the concentration profile decreases by increasing the thermal radiation parameter $\left(N_{r}\right)$ and increases by increasing Prandtl number $\left(P_{r}\right)$ and Heat source/sink parameter $(Q)$. It is also illustrated from Fig.5(d)-(e) that the concentration distribution increases with an increase of Soret number $\left(S_{r}\right)$ and Schmidt number $\left(S_{c}\right)$.

Fig.6 illustrates the variation of axial pressure gradient for different values of magnetic field parameter $(\mathrm{M})$, coupling number $(\mathrm{N})$, micropolar parameter $(\mathrm{m})$, slip parameter $(\beta)$, inclination angle $(\Theta)$ of magnetic field and the permeability parameter $\left(D_{a}\right)$. It is observed from Fig.(6) that the pressure gradient is relatively small in the wider part of the channel. 
Therefore the flow can easily pass without applying large pressure gradient. Large pressure gradient is required in the narrow part of the channel to maintain the constant flow rate to pass through it. From Fig.6(a)-(d), it is observed that the pressure gradient decreases by increasing magnetic field parameter, coupling number, micropolar parameter and slip parameter. Fig.6(e)-(f) illustrates that the inclination angle of the magnetic field and permeability parameter behavior on pressure gradient is exactly reverse when compared with the magnetic field parameter, coupling number and slip parameter. That is, the pressure gradient increases by increasing $\Theta$ and $D_{a}$.

Fig.7(a)-(f) illustrates the variation of pressure rise with respect to the non dimensional volumetric flow for different values of magnetic field parameter, coupling number, slip parameter, micropolar parameter, permeability parameter and inclination angle of the magnetic field. The region in which $\Delta p>0, \widetilde{Q}>0$ ) is known as the peristaltic pumping region. In this region the peristaltic wave overwhelms the pressure rise and propagates the fluid in the direction of its propagation. The region in which $\Delta p>0$ and $\widetilde{Q}<0$ is called a retrograde pumping region.In this locale, the stream is inverse to the course of the peristaltic movement. The region in which $\Delta p<0, \widetilde{Q}>0$ is known as augmented pumping region or co-pumping region. In this region, the negative pressure rise enhances the flow due to the peristalsis of the walls. In the free pumping region, where $\Delta p=0$, the flow is totally prompted by the peristalsis of the walls. It is observed that the pressure rise is positive below the critical value of $q$ and negative above the critical value. The critical value of $\mathrm{q}$ is denoted by $q_{0}$ and corresponds to the free pumping flow i.e. the flow when $\Delta p=0$. The effect of magnetic field parameter on the pumping phenomenon is illustrated in Fig.7(a). The pressure rise increases with an increase in $\mathrm{M}$ in the retrograde pumping region while an opposite trend is observed in other three regions. Fig.7(b)-(d) illustrates that pressure rise decreases in all the regions by increasing coupling number, slip parameter as well as micropolar parameter. From Fig.7(e)-(f), it is illustrated that that the permeability parameter and inclined magnetic field parameter show opposite behavior on pressure rise when compared with magnetic field parameter. Fig.8(a)-(f) reveals that that the frictional forces have exactly opposite behavior when compared with pressure rise. 


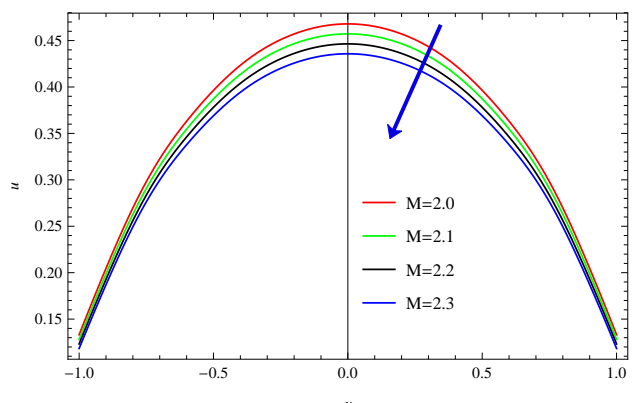

(a)

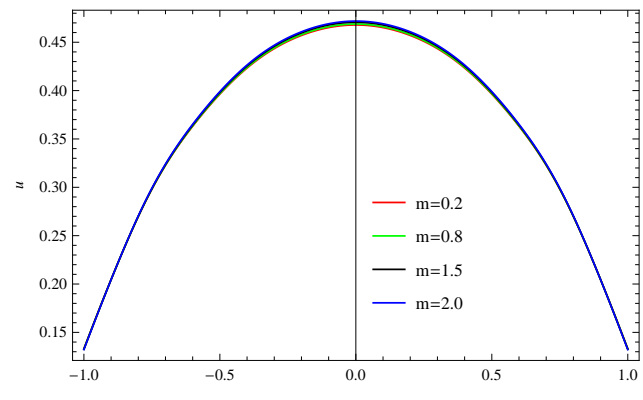

(c)

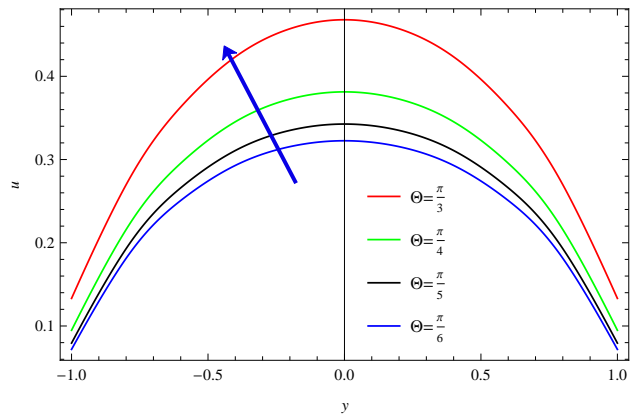

(e)

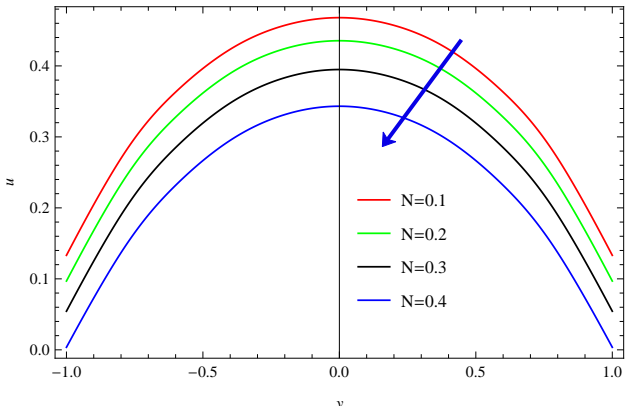

(b)

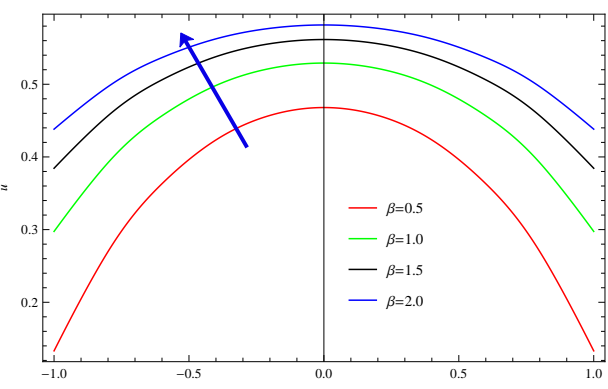

(d)

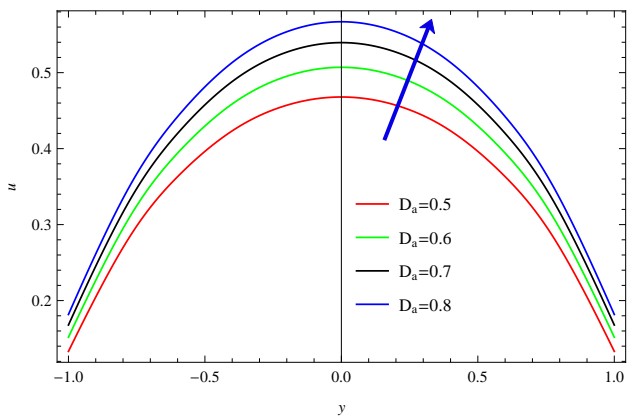

(f)

Fig. 2: Variation of $M, N, m, \beta, \Theta$ and $D_{a}$ on the axial velocity $(u)$ with respect to y with $\theta=5^{\circ}, \frac{d p}{d x}=-2, \phi=0.5, x=1, \delta=0.25, q=1$ 


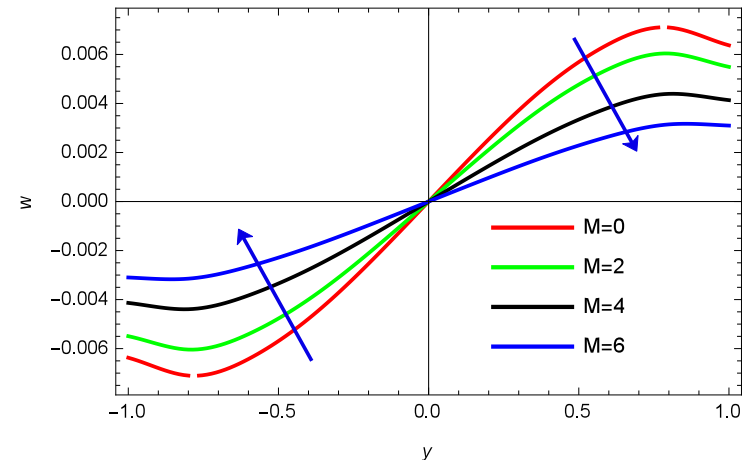

(a)

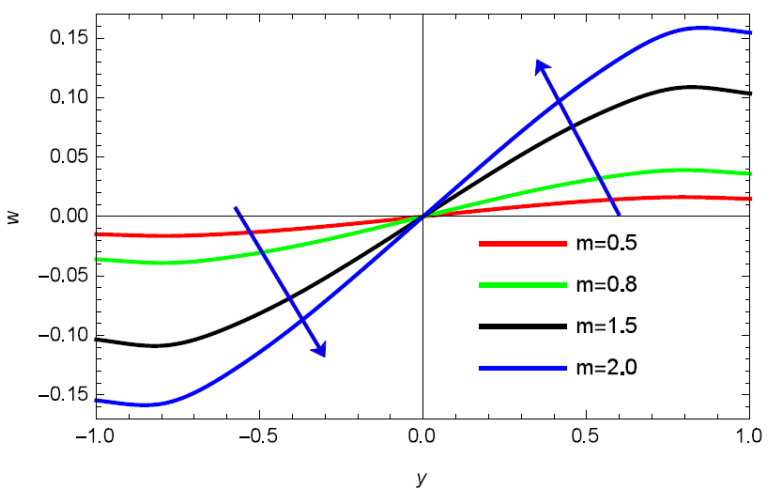

(c)

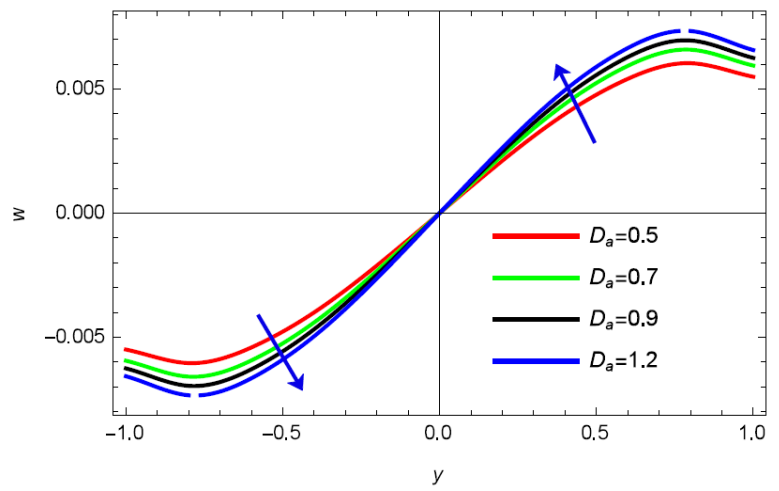

(e)

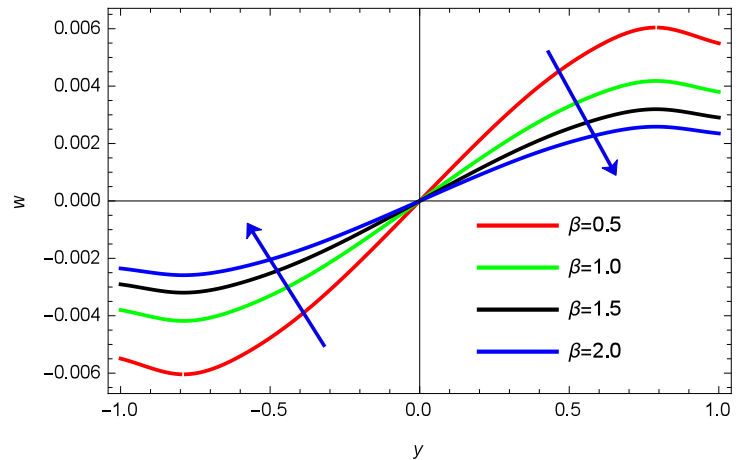

(b)

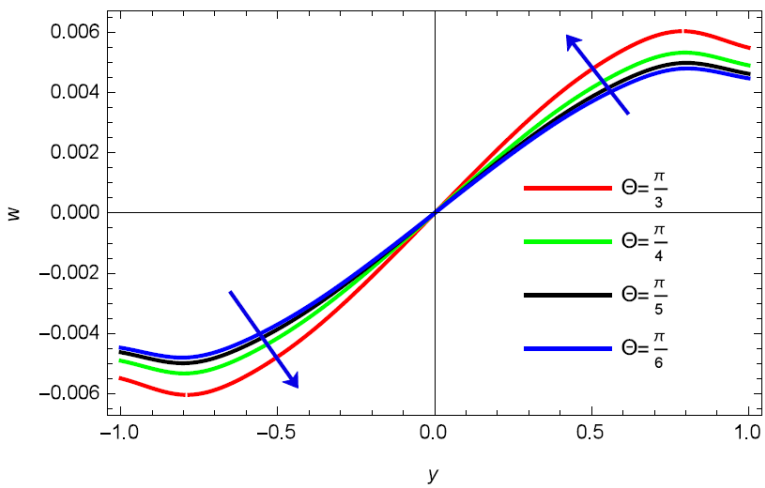

(d)

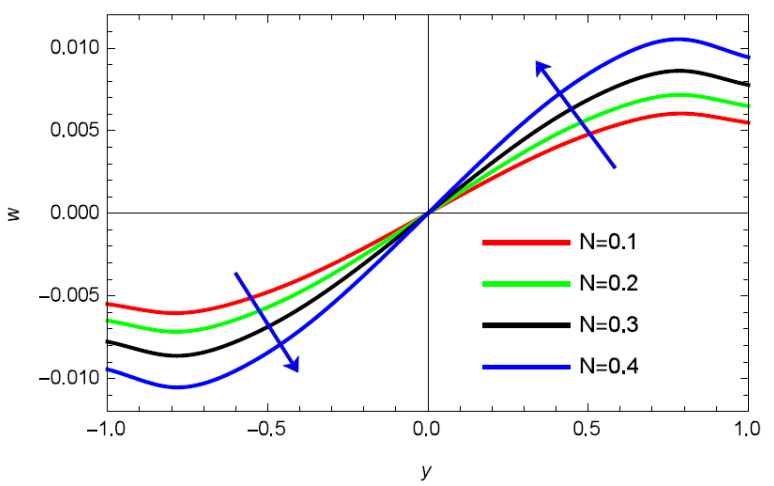

(f)

Fig. 3: Variation of $M, \beta, m, \Theta, D_{a}$ and $N$ on the spin velocity $(g)$ with respect to y $\theta=5^{\circ}, \frac{d p}{d x}=-2, \phi=0.5, x=1, \delta=$ $0.25, q=1$. 


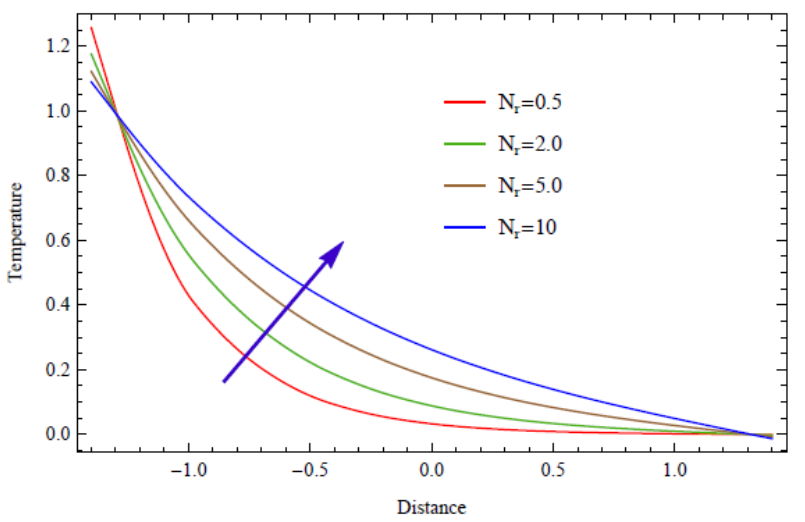

(a)

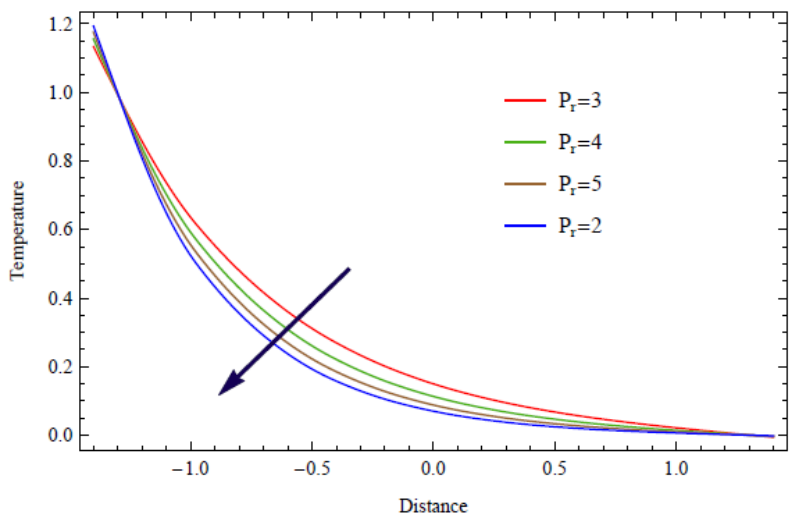

(b)

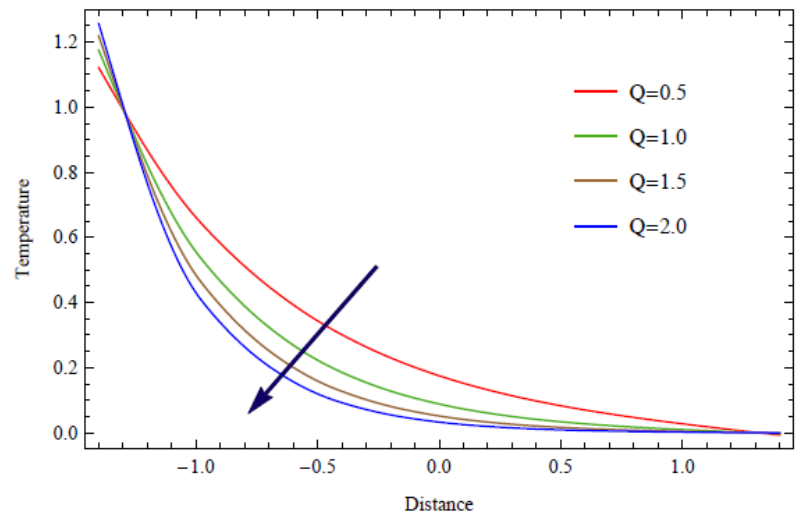

(c)

Fig. 4: Variation of $N_{r}, P_{r}$ and $Q$ on the Temperature distribution $(\theta)$ with respect to y with $\theta=5^{\circ}, \phi=0.5, x=1, \delta=0.1$ 


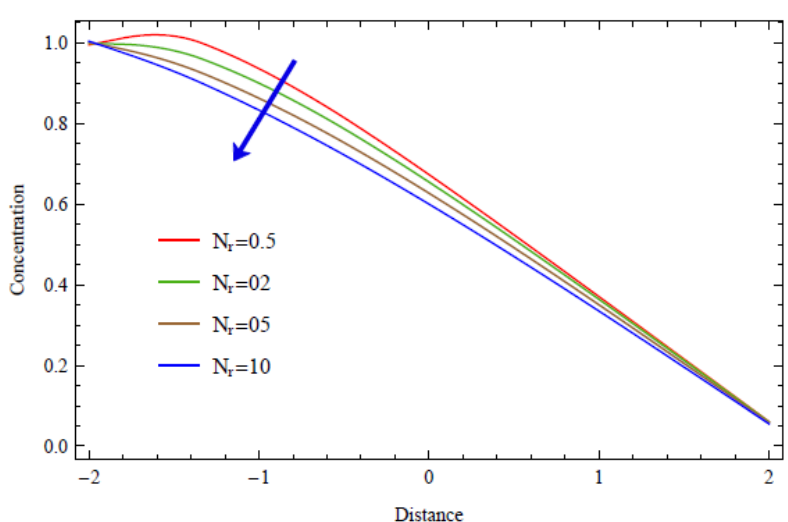

(a)

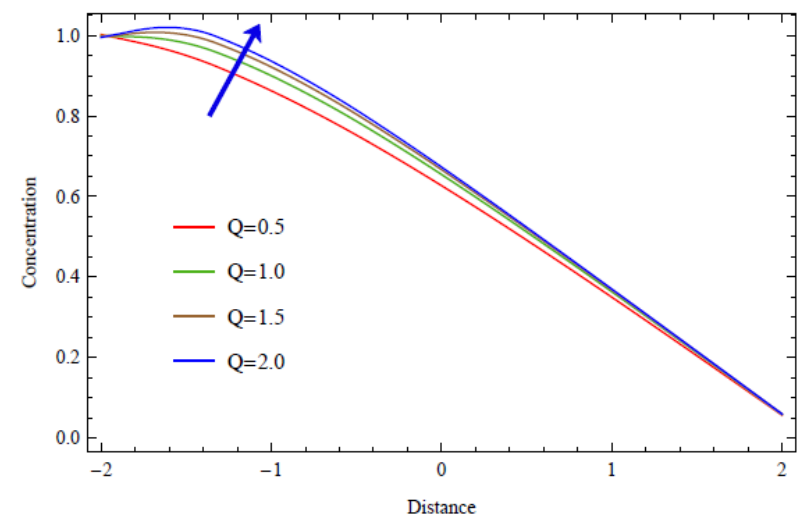

(c)

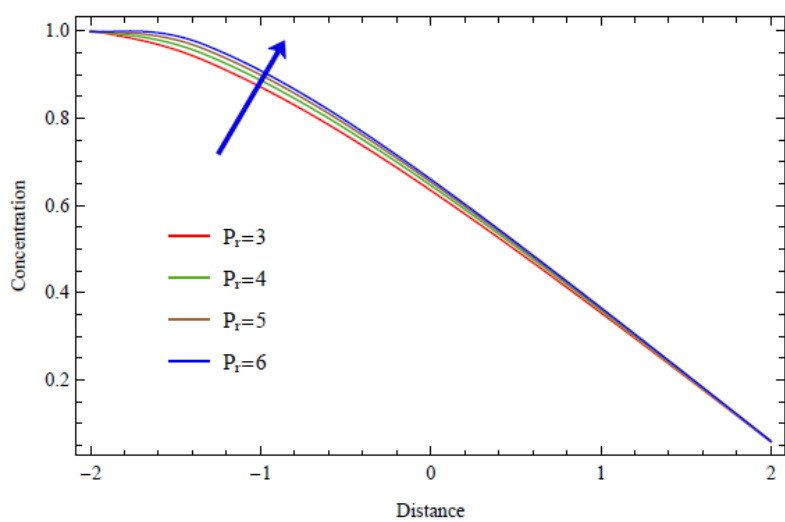

(b)

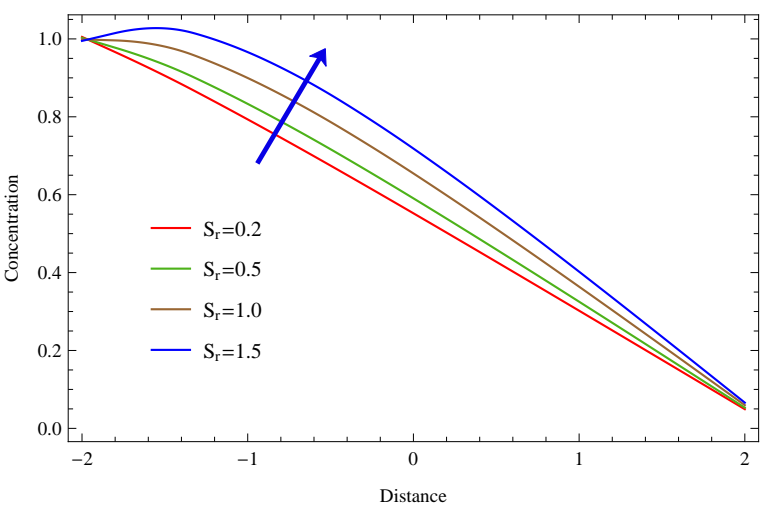

(d)

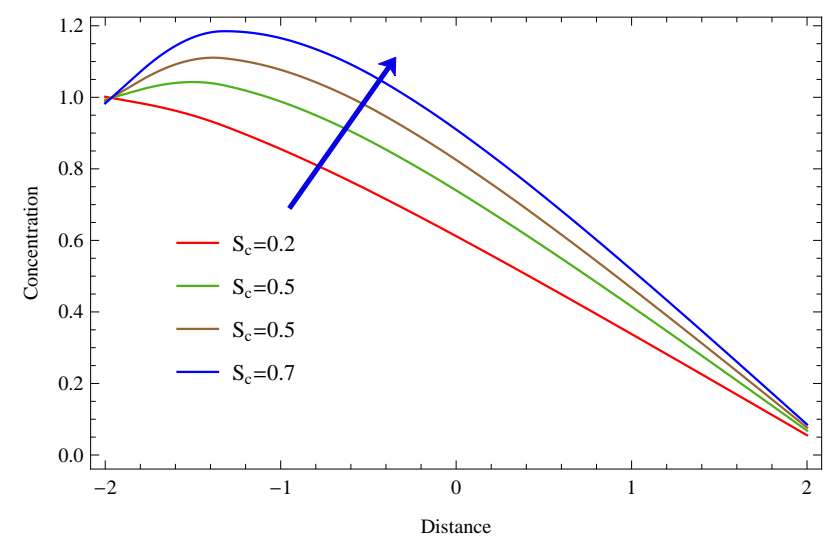

(e)

Fig. 5: Variation of $N_{r}, S_{c}, S_{r}, P_{r}$ and $S_{r}$ on the concentration profile $(\phi)$ with respect to y with $\theta=5^{\circ}, \phi=0.5, x=1, \delta=0.1$ 
240 BISK

A. A. Dar and K. Elangovan: Effect of inclined magnetic field, heat generation/absorption...

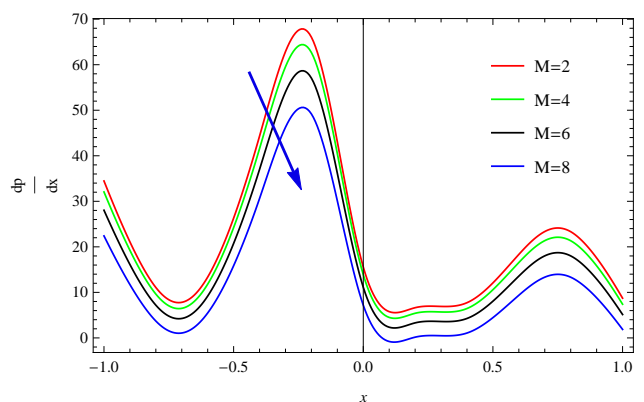

(a)

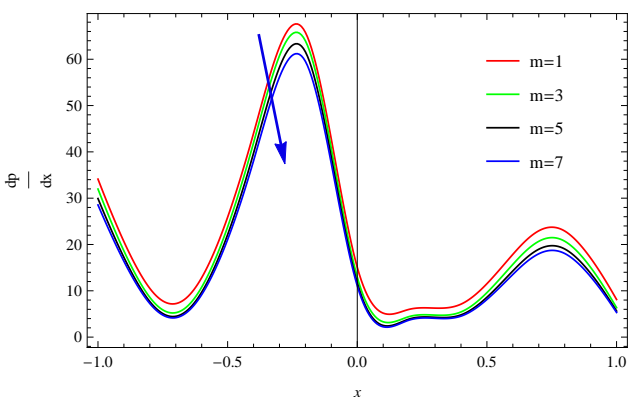

(c)

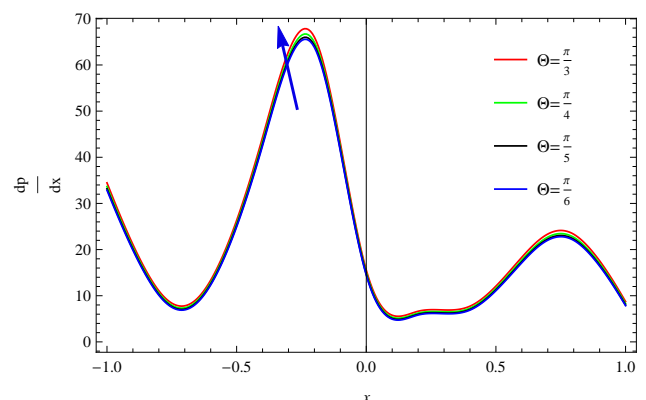

(e)

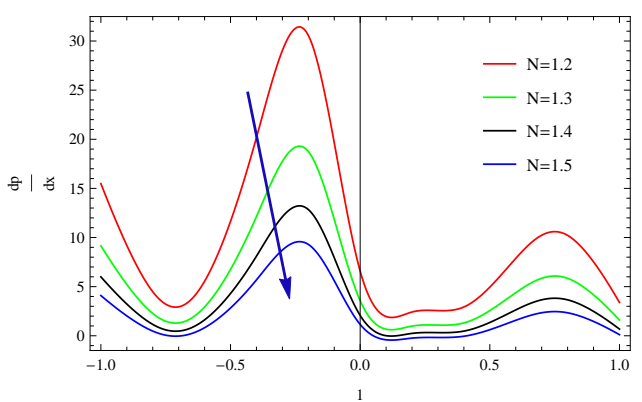

(b)

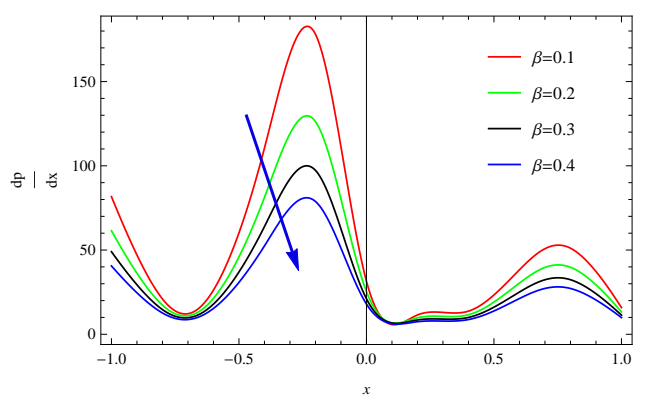

(d)

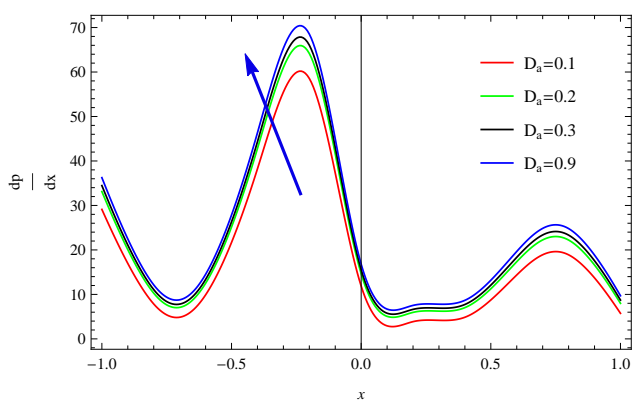

(f)

Fig. 6: Variation of $M, N, m, \beta, \Theta$ and $D_{a}$ on the pressure gradient $\left(\frac{d p}{d x}\right)$ with respect to y with $\theta=5^{\circ}, \phi=0.5, x=1, \delta=0.25$

(c) 2017 BISKA Bilisim Technology 


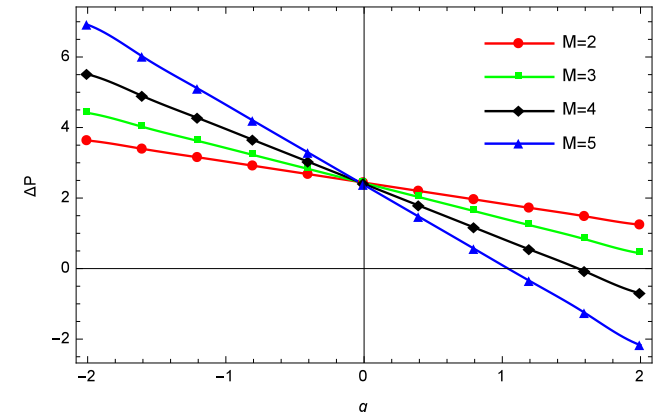

(a)

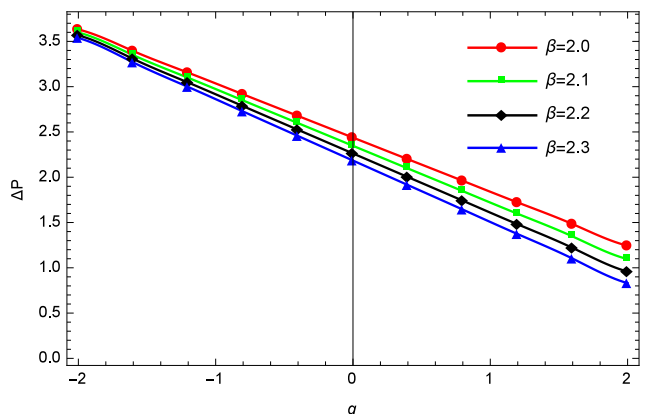

(c)

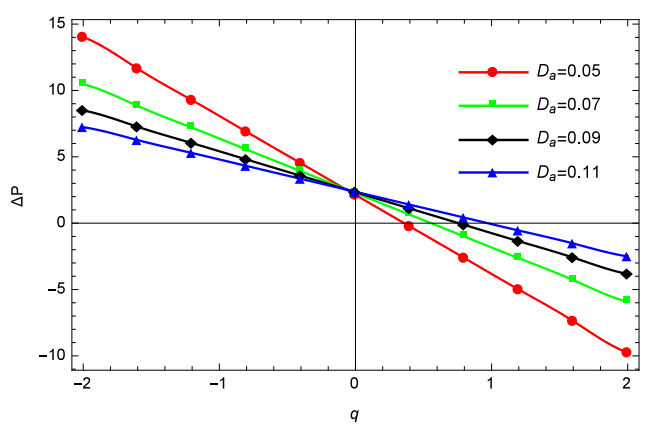

(e)

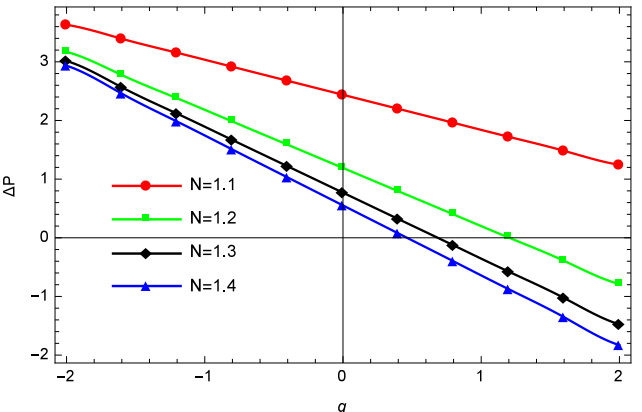

(b)

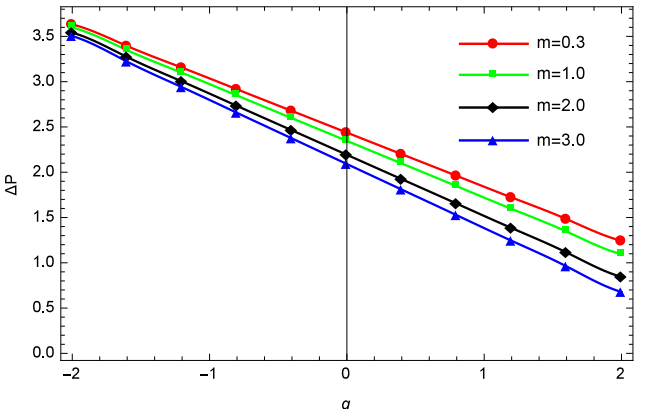

(d)

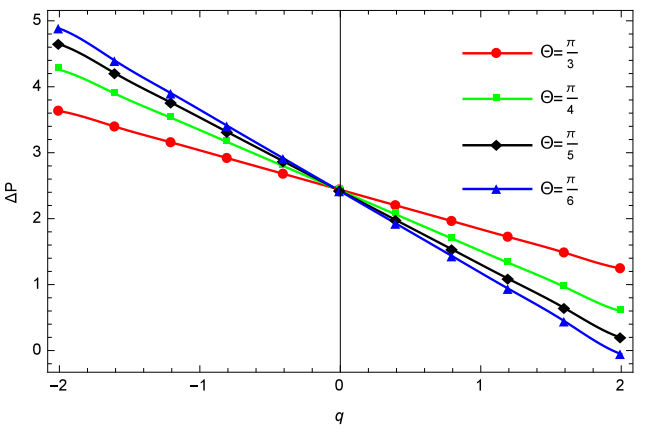

(f)

Fig. 7: Variation of $M, N, m, \beta, D_{a}$ and $\Theta$ on the pressure rise $(\Delta p)$ with respect to y with $\theta=5^{\circ}, \phi=0.5, x=1, \delta=0.25$ 


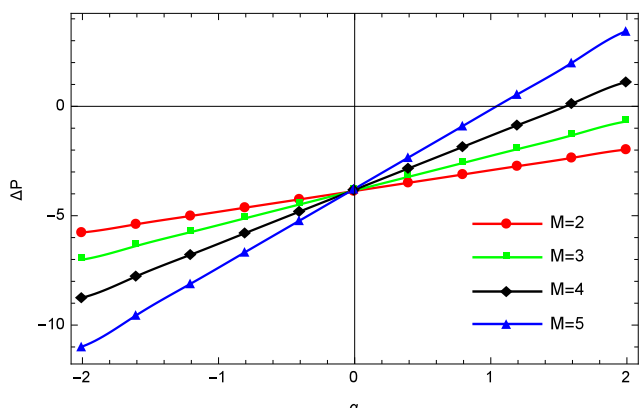

(a)

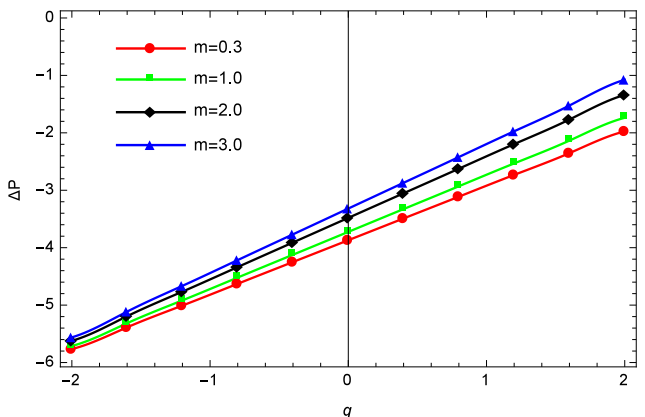

(c)

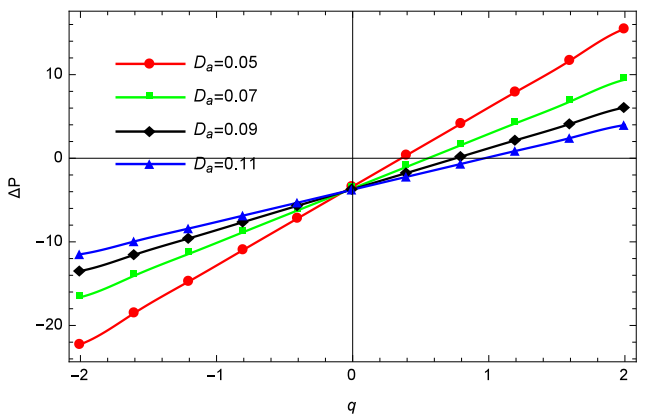

(e)

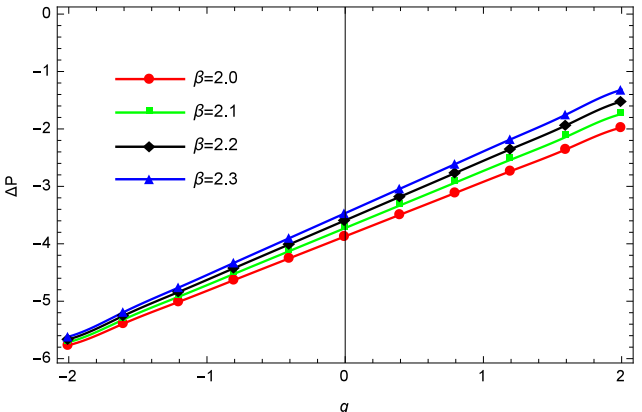

(b)

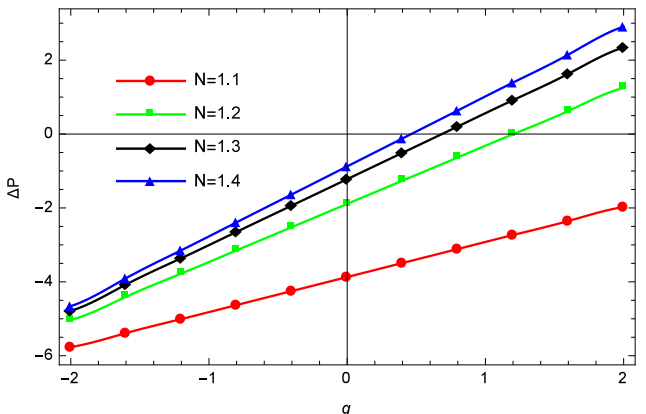

(d)

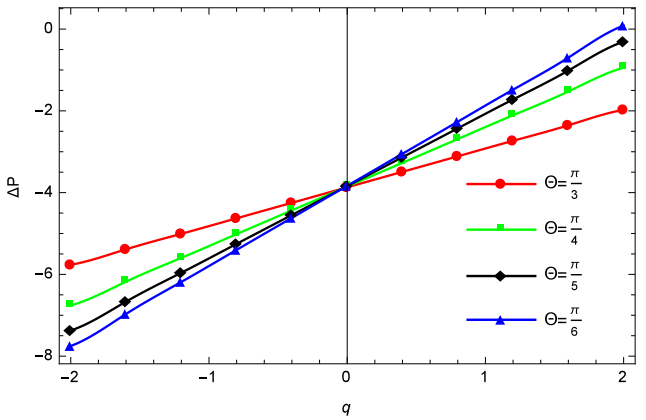

(f)

Fig. 8: Variation of $M, N, m, \beta, D_{a}$ and $\Theta$ on the pressure gradient $(F)$ with respect to y with $\theta=5^{\circ}, \phi=0.5, x=1, \delta=0.25$ 


\section{Conclusion}

In present article, an attempt has been made to examine the effects of inclined magnetic field, thermal radiation, heat source/sink on the peristaltic flow of a micropolar fluid in a porous non-uniform channel with slip velocity. To solve highly non linear differential equations, an assumption of low Reynolds number and long wavelength approximations are utilized. The key findings are enumerated below:

(i) By increasing the magnetic field parameter and the coupling number, the axial velocity and pressure gradient decreases. It is also observed that the spin velocity increases in the lower part of the channel while an opposite trend is observed in the upper part of the channel.

(ii) By increasing the slip parameter and inclination angle of the magnetic field parameter, the axial velocity increases. It specifies that the flow velocity increases from horizontal to the vertical channel.

(iii) The behavior of pressure rise is exactly opposite in comparison with frictional forces.

(iv) By increasing the Thermal radiation parameter, the magnitude of temperature distribution enhances while the value of concentration decreases.

(v) By increasing Heat sink parameter and Prandtl number, temperature shows depression while it accelerates the concentration.

(vi) By increasing Soret number and Schmidt number, the magnitude of concentration elevates.

\section{Competing interests}

The authors declare that they have no competing interests.

\section{Authors' contributions}

All authors have contributed to all parts of the article. All authors read and approved the final manuscript.

\section{References}

[1] T.W. Latham, Fluid Motion in a Peristaltic Pump, MIT, Cambridge, MA, 1966.

[2] A.H. Shapiro, M.Y. Jaffrin, S.L. Weinberg, Peristaltic pumping with long wavelengths at low Reynolds number, J. Fluid Mech. 37 (1969) 799-825.

[3] O. Eytan , A.J. Jaffa , D. Elad, Peristaltic flow in a tapered channel: Application to embryo transport within the uterine cavity. Med. Eng. Phys. 23 (2001) 473-482.

[4] M. Elshahed, M.H. Haroun, Peristaltic transport of JohnsonSegalman fluid under effect of a magnetic field, Math. Problems Eng. 6 (2005) 663-667.

[5] T. Hayat, Y. Wang, A.M. Siddiqui, K. Hutter, S. Asghar, Peristaltic transport of a third order fluid in a circular cylindrical tube, Math. Models Methods Appl. Sci. 12 (2002) 1691-1706.

[6] Kh.S. Mekheimer, Peristaltic transport of blood under effect of a magnetic field in a non-uniform channels, Appl. Math. Comput. 153 (2004) 763-777.

[7] E.F. El Shehawey, Kh.S. Mekheimer, Couple-stresses in peristaltic transport of fluids, J. Phys. D Appl. Phys. 27 (1994) $1163-1170$.

[8] Y. Wang, T. Hayat, K. Hutter, Peristaltic flow of a JohnsonSegalman fluid through a deformable tube, Theor. Comput. Fluid Dyn. 21 (2007) 369-380.

[9] Kh. S. Mekheimer, Nonlinear peristaltic transport through a porous medium in an inclined planar channel, J. Porous Media 6 (2003) 189-201. 
[10] M. Kotkandapani, S. Srinivas, Nonlinear peristaltic transport of a Newtonian fluid in an inclined asymmetric channel through a porous medium, Phys. Lett. A 372 (2008) 1265-1276.

[11] M. kothandapani, S. Srinivas, Peristaltic transport of a Jeffrey fluid under the effect of magnetic field in an asymmetric channel, Int. J. Non-Linear Mech. 43 (2008) 915-924.

[12] D. Tripathi, S.K. Pandey, S. Das, Peristaltic flow of viscoelastic fluid with fractional Maxwell model through a channel, Appl. Math. Comput. 215 (2010) 3645-3654.

[13] A. Ebaid, A new numerical solution for the MHD peristaltic flow of a biofluid with variable viscosity in circular cylindrical tube via adomian decomposition method, Phys. Lett. A 372 (2008) 5321-5328.

[14] P. Hariharan, V. Seshadri, R.K. Banerjee, Peristaltic transport of non-Newtonian fluid in a diverging tube with different wave forms, Math. Comput. Model. 48 (2008) 998-1017.

[15] M.H. Haroun, Effect of Deborah number and phase difference on peristaltic transport of a third-order fluid in an asymmetric channel, Commun. Nonlinear Sci. Numer. Simul.12 (2007) 1464-1480.

[16] A.C. Eringen, Theory of micropolar fluids, J Math Mech 16 (1966) 1-16.

[17] T. Ariman, M.A. Turk, N.D. Sylvester, Review article applications of microcontinuum fluid mechanics, Int. J. Eng. Sci. 12 (1974) 273-293.

[18] G. Lukaszewicz, Micropolar Fluids-Theory and Applications, Birkhauser, 1999.

[19] T. Hayat, N. Ali, Effects of an endoscope on the peristaltic flow of a micropolar fluid, Math. Comput. Model. 48 (2008) $721-733$.

[20] R.S. Agarwal, C. Dhanapal, Numerical solution of free convection micropolar fluid flow between two parallel porous vertical plates. Int J Eng Sci., 26 (1988) 1247-1255.

[21] M. Sheikholeslami, M. Hatami, D.D. Ganji, Micropolar fluid flow and heat transfer in a permeable channel using analytical method. J. Mol. Liq. 194 (2014) 30-36.

[22] R. Bhargava, L. Kumar, H.S. Takhar, Finite element solution of mixed convection microploar fluid driven by a porous stretching sheet, Int. J. Eng. Sci. 41 (2003) 2161-2178.

[23] M.A.A. Mahmood, S.E. Waheed, MHD flow and heat transfer of a micropolar fluid over a stretching surface with heat generation(absorption) and slip velocity, J. Egypt. Math. Soc. 20 (1) (2012) 20-27.

[24] M.M. Rahman, M.A. Sattar, Magnetohydrodynamic convective flow of a micropolar fluid past a continuously moving porous plate in the presence of heat generation/absorption. ASME J. Heat Trans. 128 (2006) 142-152.

[25] N.A. Yacos, A. Ishak, I. Pop, Melting heat transfer in boundary layer stagnation-point flow towards a stretching/shrinking sheet in a micropolar fluid, Comput. Fluids 47 (1) (2011) 16-21.

[26] H. Rosali, A. Ishak, I. Pop, Micropolar fluid flow towards a stretching/shrinking sheet in a porous medium with suction, Int. Commun. Heat Mass Transf. 39 (6) (2012) 826-829.

[27] J.C. Umavathi, J. Sultana, Mixed convective flow of a micropolar fluid mixture in a vertical channel with boundary conditions of the third kind. J Eng Phys Thermophys 85 (2012) 895-908.

[28] S.S. Motsa, S. Shateyi , The effects of chemical reaction, Hall, and ion-slip currents on MHD micropolar fluid flow with thermal diffusivity using a novel numerical technique. J Appl Math 2012 (2012) 1-30.

[29] B. Mohanty, S.R. Mishra, H.B. Pattanayak, Numerical investigation on heat and mass transfer effect of micropolar fluid over a stretching sheet through porous media, Alexandria Engineering Journal 54 (2015) 223-232.

[30] M.A. El-Aziz, Mixed convection flow of a micropolar fluid from an unsteady stretching surface with viscous dissipation, J. Egypt. Math. Soc. 21 (3) (2013) 385-394.

[31] E.A. Ashmawy, Fully developed natural convective micropolar fluid flow in a vertical channel with slip, Journal of the Egyptian Mathematical Society 23 (2015) 563-567

[32] R. Ellahi, S.U. Rahman, S. Nadeem, Noreen Sher Akbar, Influence of heat and mass transfer on micropolar fluid of blood flow through a tapered stenosed arteries with permeable walls. J Comput Theor Nanosci. 11 (2014) 1156-1163.

[33] B.I. Olajuwon, J.I. Oahimire, M. Ferdow, Effect of thermal radiation and Hall current on heat and mass transfer of unsteady MHD flow of a viscoelastic micropolar fluid through a porous medium, Engineering Science and Technology, an International Journal 17 (2014) 185-193. 